\title{
EVALUACIÓN SENSORIAL DE FRIJOL (Phaseolus vulgaris L.) ME- JORADO NUTRICIONALMENTE EN DOS COMUNIDADES CUBANAS ${ }^{1}$
}

\author{
Robert Manuel Leyva-Martínez², Helena Pachón ${ }^{3}$, Orlando Chaveco-Pérez', Nénsida Permuy-Abeleira², \\ Yovanis Ferraz-Tellez ${ }^{2}$, Naudy Caballero-Espinosa ${ }^{2}$, Evelio García-Sánchez ${ }^{2}$
}

\section{RESUMEN}

Evaluación sensorial de frijol (Phaseolus vulgaris L.) mejorado nutricionalmente en dos comunidades cubanas. El objetivo del presente trabajo fue evaluar sensorialmente el frijol común mejorado nutricionalmente (línea 38) y la variedad local (BAT 304) con productores y consumidores de dos comunidades de Holguín, Cuba. Se aplicó una encuesta exploratoria que determinó el alto consumo de los frijoles negros pequeños, y las recetas: arroz con frijoles y potaje. Se seleccionó una muestra de 80 personas y se aplicaron tres pruebas entre marzo y junio del 2008: discriminatoria triangular, aceptabilidad y preferencia. Se encontró que 50 personas discriminaron correctamente entre las dos variedades $(\mathrm{P}<0,000001)$. No hubo diferencia significativa $(\mathrm{P}=0,9)$ en el porcentaje de participantes que clasificaron (en una escala de uno a cuatro) la consistencia del caldo, el sabor del potaje y la textura del grano entre las variedades; sin embargo, al calificar estas características con palabras, notaron una diferencia en consistencia y textura, mas no en el sabor del potaje elaborado con los dos tipos de frijol. Finalmente, siete evaluadores no indicaron una preferencia por el potaje elaborado con uno u otro tipo de frijol. De los 73 que sí expresaron una preferencia, no manifestaron diferencia estadísticamente importante $(\mathrm{P}=0,13)$. En conclusión, existió diferencia sensorial entre los frijoles, pero la preferencia fue similar, lo que sugiere una buena aceptación del consumidor por el frijol mejorado nutricionalmente.

Palabras claves: Evaluación organoléptica, aceptabilidad de comida, preferencia del consumidor.

\begin{abstract}
Sensory evaluation of nutritionally improved beans (Phaseolus vulgaris L.) in two Cuban communities. The objective of this study was to evaluate the sensory characteristics of nutritionally improved common bean (line 38) and a local variety (BAT 304) among bean producers and consumers in Holguín, Cuba. An exploratory questionnaire revealed that small-seeded black beans were the most consumed, and that rice and bean casserole were the main recipes. Three sensory tests were applied to 80 individuals from March to June 2008: triangle discrimination, acceptability, and preference. Fifty participants correctly discriminated between the two bean varieties $(\mathrm{P}<0.000001)$. There was no statistical difference $(\mathrm{P}=0.9$, on a scale of 1 to 4 ) in the number of participants who classified broth consistency, the taste of the casserole, and the texture of the beans. However, when words were used to describe and score these characteristics, there was a difference between the bean varieties in consistency and texture, but not in broth taste. Seven participants indicated no preference for the casserole prepared with either bean. Of the remaining 73 that expressed a preference, there was no statistical difference $(\mathrm{P}=0.13)$ in the proportion who preferred any bean type. In conclusion, there are sensory differences between the nutritionally improved and locally consumed beans, but preference was similar, which suggests good consumer acceptance of nutritionally improved beans.
\end{abstract}

Key words: Organoleptic evaluation, food acceptability, consumer preference.

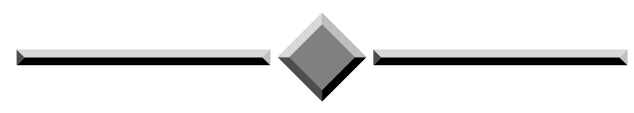

\footnotetext{
Recibido: 30 de noviembre, 2009. Aceptado: 18 de noviembre, 2010. Este trabajo forma parte del proyecto AgroSalud en Cuba, pero además constituye un capítulo de una tesis de Maestría en Ciencias Agrícolas.

2 Unidad de Extensión, Investigación y Capacitación Agropecuaria de Holguín (UEICA-H). La División, Velasco. Código postal 80144, Provincia Holguín, Cuba.

3 Centro Internacional de Agricultura Tropical (CIAT), AA 6713, Cali, Colombia. Autor encargado de la correspondencia: h.pachon@cgiar.org
} 


\section{INTRODUCCIÓN}

El frijol común (Phaseolus vulgaris L.) es entre las leguminosas de granos alimenticias, la especie más importante para el consumo humano. Su producción abarca diversas áreas; se afirma con propiedad que prácticamente se cultiva en todo el mundo (Voysest 2000).

La deficiencia de hierro es la carencia nutricional más extendida en Cuba (Reboso et al. 2002). El factor alimentario predomina en su causalidad. Alrededor del $50 \%$ de los lactantes entre seis y once meses de edad padecen de deficiencia de hierro, el 30\% de los niños de uno a tres años son anémicos, y en los niños con edad mayor de 36 meses, el hierro de la dieta y el estado de nutrición de hierro suelen ser adecuados (Rodríguez et al. 2002). Con la finalidad de prevenir la anemia durante el embarazo, desde 1993 comienza en Cuba el programa de suplementación con Prenatal (tabletas que contienen ácido fólico, fumarato ferroso), observándose que su utilización en la atención primaria, permitió disminuir en un 10\% la frecuencia de anemia en gestantes de La Habana (Padrón 1995). "Prenatal" se mantiene en ejecución y contribuye a la reducción progresiva de la prevalencia de anemia en el país.

Desde el año 2005 en Cuba se evaluó un vivero de altos minerales (VAM), con un grupo de 49 líneas de frijol común (Phaseolus vulgaris L.) mejoradas genéticamente por fitomejoramiento convencional desarrollado en el Centro Internacional de Agricultura Tropical (CIAT) y difundidas a través del proyecto AgroSalud (www.AgroSalud.org). Estos materiales contienen $33 \%$ más hierro y $17 \%$ más zinc que el frijol común que se consume actualmente en Cuba (Chaveco 2009). Estas líneas mejoradas nutricionalmente, fueron evaluadas agronómicamente durante tres años en la estación experimental de granos de la Unidad de Extensión, Investigación y Capitación Agropecuarias de Holguín (UEICA-H), en cuatro provincias cubanas $\mathrm{y}$ en diferentes formas productivas.

La calidad de un alimento está determinada por diferentes aspectos como cantidad y biodisponibilidad de nutrientes (Pachón et al. 2009) y seguridad sanitaria. Sin embargo, lo que determinará la aceptación o rechazo del mismo está relacionado con la percepción subjetiva del consumidor, es decir, aspectos ligados a la preferencia como color, sabor, textura, consistencia y presentación del producto (Liria 2007). Por esto es importante que al introducir un alimento al mercado o cambiar algún aspecto del mismo, se realicen pruebas sensoriales al grupo al cual va dirigido el alimento. El objetivo de estudio fue la evaluación sensorial de un frijol común mejorado nutricionalmente y una variedad local.

\section{MATERIALES Y MÉTODOS}

\section{Principios éticos}

El estudio se desarrolló teniendo en cuenta los siguientes principios de la declaración de Helsinki (AMM 1964). A los participantes se les comunicó el objetivo de la investigación, se les brindó información acerca del origen de los materiales, se consideró su participación voluntaria, se les informó sobre los métodos y procedimientos a seguir durante la evaluación, los beneficios futuros que tendría el consumo de los nuevos materiales de frijol mejorado nutricionalmente y como no representaba ningún riesgo para la salud de los evaluadores. En la elaboración de los formatos estuvo presente el médico de la comunidad en estudio.

\section{Ubicación del estudio}

La evaluación sensorial se realizó con productores y consumidores de las Cooperativas de Créditos y Servicios (CCS) Abel Santamaría, de la comunidad Las Caobas, y CCS Aracelio Iglesias de la comunidad Cuatro Caminos, todas del municipio Gibara, provincia Holguín. Este limita al norte con el océano Atlántico y la provincia Las Tunas, al sur con el municipio de Holguín, al este con el de Rafael Freyre y al oeste con la provincia Las Tunas y el municipio Calixto García. La extensión territorial es de $630 \mathrm{~km}^{2}$ y posee 16731 ha cultivables (Ojeda 1997). Desde el punto de vista agronómico, en ambas comunidades se evaluó un ensayo con las líneas de frijol pertenecientes al vivero de altos minerales (VAM), así como los testigos locales.

\section{La comunidad de Las Caobas}

Las Caobas es una comunidad de pobre y difícil infraestructura vial que no favorece la dinámica de comercialización agropecuaria, con limitaciones en 
la obtención de agua potable y de riego. El régimen pluviométrico se caracteriza por tener dos períodos lluviosos (mayo-junio y octubre-noviembre) y dos períodos secos (diciembre-abril y julio-septiembre) (Ojeda 1997). El promedio de precipitaciones anuales es de $1010 \mathrm{~mm}$, lo que obliga a definir bien las épocas de siembra y los cultivos correspondientes. Esto genera un uso intensivo de la tierra desde el período octubre-febrero. En este territorio no existen ríos y las fuentes de abastecimiento de agua son subterráneas, por lo que se explotan limitadamente en la irrigación agrícola por su alto contenido de sales.

\section{La comunidad de Cuatro Caminos}

En esta zona prevalecen las mejores condiciones para el desarrollo de los granos y se concentran las mayores áreas bajo riego. Es una zona de fácil acceso, lo que favorece la comercialización de los productos; y gran disponibilidad de agua, lo que permite un incremento de las áreas sembradas y mayores volúmenes en las cosechas, constituyéndose en el principal polo de producción de granos del municipio y determinante por su volumen ${ }^{4}$.

\section{Encuesta exploratoria}

Se aplicó una encuesta exploratoria en 29 hogares, considerando la ubicación geográfica dentro de la comunidad Cuatro Caminos, para conocer las principales formas de producción y hábitos de consumo del frijol, área que dedican a este cultivo dentro de la finca, número de personas que viven en el hogar, consumo promedio anual de frijol por familia, principales variedades sembradas en los últimos cinco años, y platos elaborados con el frijol, preferencia en color y tamaño del grano. Esto permitió definir la receta, el color y el tamaño del grano que se utilizaría en el estudio sensorial.

\section{Los frijoles evaluados}

Por los resultados agronómicos y de la encuesta exploratoria, se decidió utilizar la línea 38 mejorada nutricionalmente de grano negro pequeño. Como testigo para la evaluación sensorial fue seleccionado

\footnotetext{
4 Rosales, A. 2009. Caracterización de las zonas productivas. Asociación Nacional de Agricultores Pequeños en el Municipio Gibara. Cuba. Comunicación personal.
}

el BAT 304, una variedad local de color negro y tamaño pequeño, que es sembrada y consumida por los agricultores de las comunidades.

El frijol utilizado en las pruebas provino de parcelas de productores de ambas comunidades. Los materiales evaluados son de ciclo corto para el caso de Cuba (75-80 días), la variedad local supera a la mejorada nutricionalmente en cuanto a rendimiento, pero son semejantes en cuanto a tolerancia a plagas y sequía. Sin embargo, los contenidos de hierro y zinc de la variedad local son $51 \mathrm{mg} / \mathrm{kg}$ y $22 \mathrm{mg} / \mathrm{kg}$, respectivamente, mientras que la mejorada contiene $55 \mathrm{mg} / \mathrm{kg}$ de hierro y $28 \mathrm{mg} / \mathrm{kg}$ de zinc (Beebe 2009).

\section{Selección de los participantes y formatos utilizados para la evaluación sensorial}

A través de una convocatoria comunitaria, se invitó a sus habitantes a participar en forma voluntaria del estudio, en la fecha señalada. A estas personas se les aplicaron los siguientes criterios de inclusión: ser consumidor o vecino de la CCS, haber sembrado frijol común por seis meses o más, haber elaborado productos alimenticios con frijol común, y encontrarse entre 25 y 65 años de edad. Se excluyeron aquellas personas que participaron en la adaptación de los formatos para el estudio y que tuvieran una enfermedad que impidiera el proceso de degustación. Se entrevistaron 80 personas en las dos comunidades del estudio, mínimo necesario para realizar pruebas sensoriales de este tipo (Liria 2007), entre marzo y junio del 2008.

Los formatos para la evaluación sensorial (Liria 2007) fueron adaptados a las condiciones locales por investigadores de la Unidad de Extensión, Investigación y Capacitación Agropecuaria de Holguín (UEICA-H) y personas de las comunidades. Se definieron cuatro formatos: información sociodemográfica, prueba discriminatoria triangular, prueba de aceptabilidad y preferencia. Para el diligenciamiento de los formatos, se les explicó verbalmente.

\section{Preparación de la receta}

Según la encuesta exploratoria, el arroz con frijoles es la receta más utilizada. Sin embargo, se decidió preparar un potaje de frijoles para la evaluación sensorial, debido a que en esta receta el frijol es el ingrediente principal. El potaje fue probado con antelación para ajustar todos los ingredientes y ésto permitió 
definir claramente su protocolo. Para $\sim 26$ porciones, la receta consiste en $735 \mathrm{~g}$ de frijol en grano seco, agua (dos litros), sal (13 g), ajo (10 g), ají (10 g), cebolla $(25 \mathrm{~g})$, comino $(1 \mathrm{~g})$, aceite de soya $(21 \mathrm{~g})$ y puré de tomate $(26 \mathrm{~g})$. La única diferencia entre las recetas fue el tipo de frijol (línea 38 y BAT 304). El peso de los ingredientes se determinó con una balanza de $5 \mathrm{~kg}$ de capacidad y con $1 \mathrm{~g}$ de precisión. En una olla de presión se adicionaron el agua y el frijol, y se pusieron a cocinar en una hornilla eléctrica hasta que los granos se hicieran papilla al contacto con los dedos. Luego se preparó un sofrito con los demás ingredientes, se añadió al frijol cocinado y se puso nuevamente a cocinar por $\sim 2$ min. En las dos casas seleccionadas para la cocción de las recetas, el potaje fue preparado por las mismas personas, y se utilizó el mismo equipamiento para que el peso de los ingredientes y la elaboración fuera estándar en las dos comunidades.

El potaje se presentó en vasos desechables y se sirvió en cada uno $\sim 5 \mathrm{~g}$. En el plato donde se pusieron los vasos con las muestras a degustar, se escribieron los códigos de tres dígitos que identificaban las muestras previamente aleatorizadas.

\section{Encuesta sociodemográfica}

Se recolectó información de cada participante relacionada con edad, sexo, raza, lugar de nacimiento, tiempo viviendo en la comunidad, nivel de escolaridad, estado civil, vínculo laboral, área cultivable en la finca y la que dedica a la producción de frijol anualmente, así como el número de personas en el núcleo familiar.

\section{Prueba discriminatoria}

Para determinar si los consumidores discriminaban entre el potaje preparado con el frijol mejorado nutricionalmente (línea 38) y la variedad local (BAT 304), se aplicó la prueba discriminatoria triangular (Lawless 1998). En ésta, se presentaron tres muestras: dos con un tipo de frijol y una elaborada con el otro. Se les pidió que indicaran cuál de las muestras era diferente.

\section{Prueba de aceptabilidad}

El grado de aceptación de la receta se determinó mediante una prueba de aceptabilidad (Lawless 1998), utilizando una escala hedónica representada por caritas con cuatro criterios (no me gusta nada, no me gusta, me gusta, me gusta mucho) (Figura 1). Se recomienda el uso de caritas en aquellos adultos con baja escolaridad (Lawless 1998); se han usado paneles de personas no capacitadas para evaluar diferentes características del alimento (Ascheri et al. 1998, Baron y Penfield 1993, Egounlety et al. 2002). En la prueba aplicada en Cuba se consideraron tres características: consistencia, sabor y textura. Se les pidió que describieran estas características (i.e., consistencia del caldo: espesa, aguada, cremosa; sabor: agradable, desagradable; textura de grano: cascarudo, duro, blando, cascarudo y blando) y le dieran una nota (de 1 a 5 , donde 1 significa mejor).

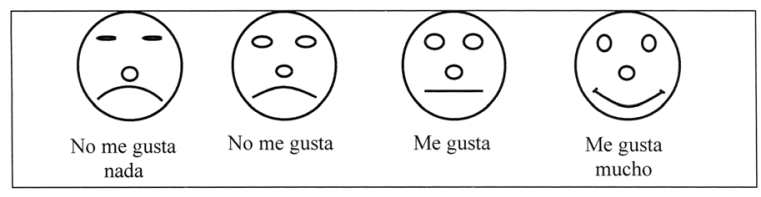

Figura 1. Escala hedónica para evaluar el grado de aceptabilidad de las recetas. Cuba, marzo a junio, 2008.

\section{Prueba de preferencia}

Al final de la evaluación se les pidió a los participantes que escogieran la muestra de potaje que preferían, ésto daba la posibilidad de seccionar a uno, a los dos o a ninguno.

\section{Análisis estadístico}

La prueba discriminatoria fue analizada mediante la prueba binomial de probabilidad, los datos de aceptabilidad con Chi-cuadrado y los de preferencia, utilizando la prueba $\mathrm{Z}$ de proporciones (Liria 2007). Estos análisis se realizaron con el programa estadístico Stata versión 9 (StataCorp LP 2005).

\section{RESULTADOS Y DISCUSIÓN}

\section{Encuesta exploratoria}

Se encuestaron 29 personas en total, 21 mujeres y ocho hombres, pertenecientes a una cooperativa de producción agropecuaria (CPA) $(n=5)$ y a una 
cooperativa de créditos y servicios (CCS) $(n=24)$. Todos los encuestados reportaron su estado civil como convivientes y tener como promedio 3,8 integrantes por núcleo familiar. Del total de los encuestados, trabajan sus fincas como productores $(n=21)$, amas de casa $(n=7)$ y trabajan fuera de la comunidad $(n=1)$. El promedio de área cultivable en las fincas que pertenecen a la CCS es de 10,9 ha y el área dedicada al frijol de 3,1 ha. En la CPA el área cultivable es de 396 ha, y dedicada al frijol de 43,2 ha. Se reportó un consumo promedio de $96,8 \mathrm{~kg}$ de frijol por año en el hogar o $25,5 \mathrm{~kg}$ de frijol percápita anual. En los últimos cinco años sembraron fundamentalmente dos variedades: Velasco largo (color rojo, tamaño mediano) y BAT 304 (color negro, tamaño pequeño); en $67,5 \%$ de las familias, la última variedad fue la que predominó. El plato preferido es la mezcla de arroz con frijoles denominada congrís $(72,5 \%)$, seguido por el frijol blando en caldo con especias, al que se nombra potaje $(27,5 \%)$; otro plato utilizado es el cocido, que es el frijol blando y seco con especias, y por último la ensalada, que es el frijol frío con cebolla blanca. Pese a que el congrís es el plato preferido y de mayor consumo, se decidió emplear el potaje en la evaluación sensorial debido a que se podían evaluar mejor las características organolépticas del frijol solo, a diferencia del que se acompaña con arroz. En el potaje, preferían el frijol rojo $(65,5 \%)$ que el negro. Sin embargo, se decidió usar el negro, debido que el rojo mejorado nutricionalmente evidenciaba una diferencia notable con el rojo testigo en cuanto a color y tamaño, lo que podría condicionar su valoración.

\section{Encuesta sociodemográfica}

Todos los participantes pertenecían a la raza blanca. El 92,5\% nacieron en la Provincia de Holguín y el $56,1 \%$ eran mujeres (Cuadro 1). El promedio de permanencia en las dos comunidades donde se realizó el estudio es de 33,6 años. En cuanto al nivel de escolaridad de los participantes se evidencian cinco niveles: nivel primario (17,5\%), nivel secundario $(36,3 \%)$, preuniversitario $(31,3 \%)$, técnico medio $(3,8 \%)$ y universitario (11,3\%). 98,7\% del total de las personas que participaron en el estudio, trabajaban, y de ellos, $16,3 \%$ están vinculados con el estado, $32,5 \%$ tienen su propia finca, $37,5 \%$ son amas de casa, $8,7 \%$ estudian y trabajan en la CPA y $3,7 \%$ son jubilados; ninguno estaba en la categoría de cuenta propia (personas que no tienen vínculo laboral con el estado y pagan un impuesto a éste por realizar trabajos privados). El área promedio cultivable es de 81,7 ha y de este total, 34,7 ha se dedican al cultivo del frijol.

\section{Discriminatoria}

De las 80 personas, 50 discriminaron correctamente que la muestra era diferente a las otras dos $(\mathrm{P}<0,000001)$.

\section{Aceptabilidad}

Según la escala hedónica de aceptabilidad, no hubo una diferencia estadísticamente significativa $(\mathrm{P}=0,9)$ en el porcentaje de participantes que clasificaron la consistencia del caldo, el sabor del potaje y la textura del grano, considerando los cuatro criterios evaluados (Cuadro 2). Por ejemplo, 55\% de los participantes marcaron "me gusta" a la consistencia del caldo de la línea 38, en comparación con $60 \%$ para el frijol BAT 304. Para las tres características, entre $54 \%$ y $63 \%$ de las personas escogieron "me gusta" para ambos frijoles. De igual manera, no hubo diferencia $(\mathrm{P} \geq 0,09)$ en la calificación que los participantes le asignaron a la consistencia, sabor y textura de los dos frijoles (Cuadro 3). Entre $59 \%$ y $68 \%$ de las personas calificaron como "buena" la consistencia, el sabor y la textura del potaje elaborado con la línea 38 y el BAT 304. Hubo una diferencia estadísticamente significativa $(\mathrm{P} \leq 0,02)$ en el porcentaje de participantes que clasificaron el potaje, según las características de consistencia del caldo y textura del grano, mas no de sabor $(\mathrm{P}=0,43)$ (Cuadro 4). Una mayor proporción $(58 \%)$ de los participantes clasificaron la consistencia del caldo del potaje elaborado con la línea 38 como "cremosa", en comparación con el BAT 304 (34\%). Se encontró lo contrario para la caracterización "espesa" de la consistencia de caldo de los frijoles (33\% para la línea 38 y $54 \%$ para el BAT 304). En cuanto a la textura del grano, $9 \%$ de los participantes clasificaron como "cascarudo" a la textura del potaje con la línea 38, en comparación con $19 \%$ para el BAT 304. En cuanto al sabor, $90 \%$ y $94 \%$ de los comensales clasificaron el sabor del potaje como "agradable" para la línea 38 y el BAT 304, respectivamente.

Un elemento importante que determinó en gran medida la aceptabilidad del frijol mejorado nutricionalmente, además de sus características organolépticas, fue el color y el tamaño del grano, pues no existía una 
Cuadro 1. Características sociodemográficas de las personas que participaron en el estudio sensorial, según comunidad. Cuba. Marzo a junio, 2008.

\begin{tabular}{|c|c|c|c|}
\hline Características & $\begin{array}{l}\text { Cuatro Caminos } \\
(n=40)\end{array}$ & $\begin{array}{l}\text { Las Caobas } \\
\quad(n=40)\end{array}$ & $\begin{array}{r}\text { Total } \\
(\mathbf{n}=\mathbf{8 0})\end{array}$ \\
\hline Edad, años & 35,8 & 42,9 & 39,3 \\
\hline Mujeres, $\%$ & 60,0 & 52,2 & 56,1 \\
\hline Permanencia en la comunidad, años & 28,1 & 39,1 & 33,6 \\
\hline \multicolumn{4}{|l|}{ Escolaridad, \% } \\
\hline Nivel primario (preescolar al $6^{\circ}$ ) & 5 & 30 & 17,5 \\
\hline Nivel secundario $\left(7^{\circ}\right.$ al $\left.9^{\circ}\right)$ & 35 & 37,5 & 36,3 \\
\hline Preuniversitario $\left(10^{\circ}\right.$ al $\left.12^{\circ}\right)$ & 35 & 27,5 & 31,3 \\
\hline Técnico $\left(10^{\circ}\right.$ al $12^{\circ}$, especializado $)$ & 5 & 2,5 & 3,8 \\
\hline Universitario & 20 & 2,5 & 11,3 \\
\hline \multicolumn{4}{|l|}{ Estado civil, \% } \\
\hline Casado & 22,5 & 32,5 & 27,5 \\
\hline Convive & 45 & 55 & 47,5 \\
\hline Separado & 2,5 & 2,5 & 2,5 \\
\hline Divorciado & 5 & 5 & 5 \\
\hline Soltero & 17,5 & 2,5 & 10 \\
\hline Viudo & 5 & 2,5 & 3,8 \\
\hline Integrantes del hogar, $\mathrm{n}$ & 3,7 & 3,2 & 3,5 \\
\hline Trabajan, $\%$ & 97,5 & 100 & 98,7 \\
\hline \multicolumn{4}{|l|}{ Lugar de trabajo, $\%$} \\
\hline Con el estado & 25 & 7,5 & 16,3 \\
\hline Cuenta propia & 0 & 0 & 0 \\
\hline En la finca & 25 & 40 & 32,5 \\
\hline Ama de casa & 30 & 45 & 37,5 \\
\hline Estudia y trabaja en la cooperativa & 12,5 & 5 & 8,8 \\
\hline Jubilado & 5 & 2,5 & 3,7 \\
\hline $\begin{array}{l}\text { Promedio de área cultivable en las fincas de los } \\
\text { encuestados, ha }\end{array}$ & 2,4 & 1,7 & 81,7 \\
\hline $\begin{array}{l}\text { Promedio de área dedicada a la producción de } \\
\text { frijol en las fincas de los encuestados, ha }\end{array}$ & 1,3 & 0,4 & 34,7 \\
\hline
\end{tabular}

diferencia notable después de preparado y, de acuerdo a la encuesta exploratoria, en los últimos cinco años el $67,5 \%$ de las familias sembraron fundamentalmente BAT 304 (color negro, tamaño pequeño).

Pese a que la aceptación de los frijoles pueda estar influenciada por hábitos de consumo y características como el color del grano, tamaño o costumbres por zonas o países, se puede plantear, para este caso, que la aceptabilidad del frijol mejorado nutricionalmente es el resultado de poseer buenas características organolépticas como espesor del caldo para el potaje, sabor y textura del grano; coincidiendo con el planteamiento de Hernández et al. (2002) sobre el usuario mexicano que desea un caldo de frijol con buena apariencia, sabor y textura.

\section{Preferencia}

De las 80 personas que participaron en el estudio, siete no indicaron una preferencia por el potaje elaborado con uno u otro tipo de frijol. De los 73 que sí expresaron una preferencia, no hubo una diferencia 
Cuadro 2. Porcentaje de evaluadores que clasificaron el potaje elaborado con dos tipos de frijol, según cuatro criterios de aceptabilidad. Cuba. Marzo a junio, 2008.

\begin{tabular}{lccccc}
\hline \multirow{2}{*}{ Característica } & \multicolumn{5}{c}{ Criterio de aceptabilidad } \\
\cline { 2 - 6 } & $\begin{array}{c}\text { No me gusta } \\
\text { nada (\%) }\end{array}$ & $\begin{array}{c}\text { No me gusta } \\
(\%)\end{array}$ & $\begin{array}{c}\text { Me gusta } \\
(\%)\end{array}$ & $\begin{array}{c}\text { Me gusta } \\
\text { mucho (\%) }\end{array}$ & $\begin{array}{c}\text { Valor P según } \\
\text { Chi-cuadrado }\end{array}$ \\
\hline Consistencia del caldo & & & & & 0,9 \\
Línea 38 & 0 & 10 & 55 & 35 & \\
BAT 304 & 0 & 9 & 60 & 31 & 0,9 \\
Sabor & 3 & 9 & 58 & 31 & \\
Línea 38 & 0 & 8 & 63 & 30 & 0,9 \\
BAT 304 & & & & & \\
Textura de grano & 0 & 11 & 54 & 35 & \\
Línea 38 & 0 & 10 & 61 & 29 & \\
BAT 304 & & & & & \\
\hline
\end{tabular}

Cuadro 3. Porcentaje de evaluadores que clasificaron de 1 (bueno) a 5 (malo) al potaje elaborado con dos tipos de frijol. Cuba. Marzo a junio, 2008.

\begin{tabular}{lcccccc}
\hline Característica & \multicolumn{6}{c}{ Criterio de aceptabilidad } \\
\cline { 2 - 7 } & $\mathbf{1}$ & $\mathbf{2}$ & $\mathbf{3}$ & $\mathbf{4}$ & $\mathbf{5}$ & $\begin{array}{c}\text { Valor P } \\
\text { según }\end{array}$ \\
& & & & & & $\begin{array}{c}\text { Chi-cua- } \\
\text { drado }\end{array}$ \\
\hline & & & & & & 0,94 \\
$(\boldsymbol{\%})$ & $(\boldsymbol{\%})$ & $\mathbf{( \% )}$ & \\
\hline $\begin{array}{l}\text { Con siste n cia } \\
\text { del caldo, \% }\end{array}$ & & & & & & \\
Línea 38 & 60 & 21 & 10 & 1 & 8 & \\
BAT 304 & 66 & 20 & 8 & 1 & 5 & \\
Sabor, \% & & & & & & 0,09 \\
Línea 38 & 68 & 25 & 6 & 1 & 10 & \\
BAT 304 & 63 & 19 & 9 & 5 & 5 & \\
Textura de & & & & & & 0,86 \\
grano, \% & & & & & & \\
Línea 38 & 66 & 18 & 9 & 3 & 5 & \\
BAT 304 & 59 & 15 & 16 & 6 & 4 & \\
\hline
\end{tabular}

estadísticamente significativa $(\mathrm{P}=0,13)$ entre los tipos de frijol: línea $38(n=33)$ y BAT $304(n=40)$. El hecho de no existir una diferencia estadísticamente significativa frente a la preferencia por cualquiera de los platos, evidencia que a pesar de las opiniones contrarias detectadas en las pruebas de discriminación y aceptabilidad, los comensales estarían dispuestos a consumir tanto la línea 38 como la variedad local BAT 304.

Esta investigación es el primer estudio sensorial que se realiza con frijoles mejorados nutricionalmente en hierro y zinc. En este trabajo se adelantaron pruebas de discriminación, preferencia y aceptabilidad, tanto para consistencia del caldo como para sabor y textura del grano, dado a que es la forma más directa

Cuadro 4. Porcentaje de evaluadores que clasificaron el potaje elaborado con dos tipos de frijol, según categorías descriptivas. Cuba. Marzo a junio, 2008.

\begin{tabular}{lccc}
\hline Características & $\begin{array}{c}\text { Línea 38 } \\
(\%)\end{array}$ & $\begin{array}{c}\text { BAT 304 } \\
(\%)\end{array}$ & $\begin{array}{c}\text { Valor P } \\
\text { según Chi- } \\
\text { cuadrado }\end{array}$ \\
\hline Consistencia del caldo & & & 0,007 \\
Espesa & 33 & 54 & \\
Aguda & 10 & 13 & \\
Cremosa & 58 & 34 & \\
Sabor & & & 0,43 \\
Agradable & 90 & 94 & \\
Desagradable & 10 & 6 & \\
Textura del grano & & & \\
Cascarudo & 9 & 19 & \\
Duro & 6 & 1 & \\
Blando & 85 & 80 & \\
\hline
\end{tabular}


de medir la calidad de un producto alimenticio, pues el hombre realiza la evaluación de sus propiedades organolépticas con sus sentidos (Espinosa 2007).

En conclusión, el frijol común mejorado nutricionalmente tuvo gran aceptación entre productores y consumidores de las dos comunidades que participaron de la evaluación, mostrando buenas características organolépticas como espesor del caldo para el potaje, sabor y textura del grano. Por consiguiente, el frijol común mejorado nutricionalmente puede convertirse en uno de los de mayor preferencia de productores y consumidores de las comunidades involucradas en el estudio; y su difusión, multiplicación y consumo puede contribuir a la solución de la deficiencia de hierro en el país.

\section{AGRADECIMIENTOS}

A María Reyna Liria Domínguez por su capacitación en metodología sensorial, al apoyo financiero del Proyecto AgroSalud (CIDA 7034161) para realizar el estudio, a Fredy Salazar por su ayuda con el análisis estadístico de los datos y a Marlene Rosero por su edición del manuscrito. Se reconoce la colaboración local de las comunidades Cuatro Caminos y Las Caobas para realizar el estudio y la del Maestro en Ciencia, Agustín Serrano Santiesteban, por el apoyo incondicional en la revisión del artículo.

\section{LITERATURA CITADA}

Ascheri, JLR; Bernal-Gómez, ME; Carvalho, CWP; Modesta, RCD. 1998. Producción de snacks, a partir de mezclas de harina de arroz y camote, por extrusión termoplástica: parte II propiedades funcionales y sensoriales. Alimentaria 293:79-86.

AMM (Asociación Médica Mundial). 1963. Principios éticos para las investigaciones médicas en seres humanos (en línea). Finlandia. Consultado 6 abril 2009. Disponible en http://www.bioetica.uchile.cl/doc/helsink.htm

Baron, RF; Penfield, MP. 1993. Panelist texture preferences affect sensory evaluation of green bean cultivars (Phaseolus vulgaris L.). Journal of Food Science 58(1):138-139.

Beebe, S. 2009. Resultados de los análisis de absorción atómica para la determinación del contenido de hierro y zinc. Muestras 2827, 2876, 2925, 2996, 3128. CIAT. Cali, Colombia. Sin publicar.
Chaveco, O. 2009. Análisis del contenido de micronutrientes de las líneas mejoradas nutricionalmente existente en Cuba y de variedades del germoplasma cubano. UEICA-H. Cuba. Sin publicar.

Egounlety, M; Aworh, OC; Akingbala, JO; Houben, JH; Nago, MC. 2002. Nutritional and sensory evaluation of tempe-fortified maize-based weaning foods. International Journal of Food Sciences and Nutrition 53:15-27.

Espinosa, J, 2007. Evaluación sensorial de los alimentos. La Habana, Cuba. Editorial Universitaria. 97 p.

Hernández, J; Hernández, H; Azpíroz, S; Acosta, J; Bernal, I. 2002. Caracterización de una población de líneas endogámicas de frijol común por su calidad de cocción y algunos componentes nutrimentales. Agrociencia 36(4):451-459.

Lawless, H. 1998. Sensory evaluation of food: principles and practices. Nueva York, USA, Kluwer Academics/Plenum Publishers. 827 p.

Liria, MR. 2007. Guía para la evaluación sensorial de alimentos. Cali, Colombia. Centro Internacional de Agricultura Tropical. $22 \mathrm{p}$.

Ojeda, FR. 1997. Métodos y experiencias en extensión agropecuaria para un desarrollo sostenible: zonificación del municipio Gibara, provincia de Holguín, Cuba. ETIA-H. 4 p.

Pachón, H; Ortiz, DA; Araujo, C; Blair, MW; Restrepo, J. 2009. Iron, zinc and protein bioavailability proxy measures of meals prepared with nutritionally enhanced beans and maize. Journal of Food Science 74(5):H147-H154.

Padrón, M. 1995. El suplemento "Prenatal" para la prevención de la anemia en el embarazo. Revista Cubana Alimentos y Nutrición 9(1):74-75.

Reboso, J; Cabrera, E; Pita, G; Jiménez, S. 2005. Anemia por deficiencia de hierro en niños de 6 a 24 meses y de 6 a 12 años de edad. Instituto de Nutrición e Higiene de los Alimentos. Revista Cubana Salud Pública 31(4):306-312.

Rodríguez, J; Pérez, J; Hernández, A; Triana, M; Chong, A; Sánchez, M. 2002. Anemia nutricional en un grupo de niños aparentemente sanos de 2 a 4 años de edad. Instituto de Nutrición e Higiene de los Alimentos. Revista Cubana Alimentos y Nutrición 16(1):31-34.

StataCorp LP. 2005. Intercooled Stata 9.0 for Windows. College Station, USA, StataCorp LP.

Voysest, O. 2000. Mejoramiento genético del frijol (Phaseolus vulgaris L.): legado de variedades de América Latina 1930-1999. Cali, Colombia, Centro Internacional de Agricultura Tropical. 195 p.

AGRONOMÍA MESOAMERICANA 21(2):281-288. 2010 